\title{
New paradigms for managing preoperative anemia
}

\author{
Andrew Verniquet, MD $\cdot$ Rafid Kakel, MD
}

Received: 29 September 2011/ Accepted: 26 October 2011/Published online: 7 January 2012

(c) Canadian Anesthesiologists' Society 2012

\section{To the Editor,}

In the Continuing Professional Development article, Assessment and treatment of preoperative anemia, Hare et al. ${ }^{1}$ present very interesting concepts and hypotheses. Despite the important concepts, implementation of the proposed protocol would inevitably result in delays as well as logistical and financial implications related to the supervised administration of intravenous iron and erythropoietic stimulating agents (ESAs). Also, it is questionable whether the evidence is sufficiently strong to incorporate the proposed protocol into routine clinical practice.

The protocol in the article appears to be based on the Network for Advancement of Transfusion Alternatives (NATA) guidelines, ${ }^{2}$ which were directed specifically toward elective orthopedic surgical patients. The authors of the NATA guidelines made five recommendations graded as strong or weak according to the risk/benefit ratio, and they classified the respective quality of evidence as high, moderate, or low/very low. There are three strong recommendations regarding the timing of testing, laboratory tests, and the treatment of nutritional deficiency anemia, yet the recommendations are based on low/very low-quality evidence. Also, a weak recommendation regarding the target hemoglobin level is based on low/very low-quality evidence. The only recommendation based on high-quality evidence is the final weak recommendation regarding the use of ESAs in anemia where nutritional deficiency has been ruled out.

The same group also produced a consensus statement ${ }^{3}$ regarding the use of intravenous iron wherein they

A. Verniquet, MD (凶) $\cdot$ R. Kakel, MD

James Paton Memorial Hospital-Central Health,

Gander, NF, Canada

e-mail: andrewverniquet@hotmail.com concluded that intravenous iron may be indicated in the perioperative period in orthopedic patients who are expected to develop severe postoperative anemia. Again, this is a weak recommendation based on moderate/ lowquality evidence, and no evidence-based recommendations could be made for other types of surgery.

With regard to the use of ESAs in cancer patients, the European Medicines Agency recommends that transfusion be the preferred method for correcting anemia in cancer patients, especially those with a long life expectancy. ${ }^{4}$ The Food and Drug Administration changed the product label for ESAs by adding a black box warning which specifies that ESAs are not indicated to treat cancer-induced anemia in patients not on chemotherapy or in patients with potentially curable cancer. These recommendations were echoed by the American Society of Clinical Oncology clinical practice guidelines. ${ }^{5}$

Competing interests None declared.

\section{References}

1. Hare GM, Baker JE, Pavenski K. Assessment and treatment of preoperative anemia: Continuing Professional Development. Can J Anesth 2011; 58: 569-81.

2. Goodnough LT, Maniatis A, Earnshaw P, et al. Detection, evaluation, and management of preoperative anaemia in the elective orthopaedic surgical patient: NATA guidelines. Br J Anaesth 2011; 106: 13-22.

3. Beris P, Munoz M, Garcia-Erce JA. Perioperative anaemia management: consensus statement on the role of intravenous iron. Br J Anaesth 2008; 100: 599-604.

4. European Medicine Agency Press Office. Press Release. EMEA Recommends a New Warning for Epoetins for Their Use in Cancer Patients. Available from URL: http://www.emea.europa. eu/docs/en_GB/document_library/Press_release/2009/11/WC500 015069.pdf (accessed October 2011). 
5. Rizzo JD, Somerfield MR, Hagerty KL, et al. Use of epoetin and darbepoetin in patients with cancer: 2007 American Society of Hematology/American Society of Clinical Oncology clinical practice guideline update. Blood 2008; 111: 25-41.

\section{Reply}

The authors appreciate the comments of Dr. Verniquet with respect to concerns about important practical aspects of anemia treatment in perioperative patients. The proposed treatment protocol is based on standard clinical approaches to the treatment of anemia associated with iron deficiency and inflammatory conditions, the most common forms of anemia in perioperative patients. These recommendations are consistent with the Network for Advancement of Transfusion Alternatives guidelines. In addition, the concerns raised about potential toxicity of therapies, including intravenous iron and erythrocyte stimulating agents, must be considered. The authors agree that care must be taken when utilizing erythropoietic stimulating agent therapy, and practitioners must be aware of the relative and absolute contraindications for its use-as addressed in our Continuing Professional Development module. When utilizing any therapy, the treatment must be individualized to the specific patient circumstances, and the potential for therapeutic benefit must always be balanced by consideration for the risk of adverse effects.

Unfortunately, there is limited prospective randomized outcome data to support the benefit of anemia treatment in cardiac and non-cardiac surgery. This may be a result of previously published reports which supported a different perspective, namely, that anemia is safe, ${ }^{1}$ adaptive, ${ }^{2}$ and therapeutic. ${ }^{3}$ However, these studies were not undertaken in patients undergoing surgery who experience hemodynamic stress associated with acute blood loss, surgical trauma, and inflammation. An increasing number of clinical studies have shown that acute anemia is an independent predictor for increased mortality in this patient population. The magnitude of this problem is emphasized by two recently published retrospective studies which demonstrate that up to $30-50 \%$ of our patients suffer from preoperative anemia and that even mild to moderate anemia is associated with significant increases in perioperative mortality. ${ }^{4,5}$ In addition, a newly published experimental study emphasizes that these adverse clinical outcomes likely occur through complex mechanisms that include both hypoxic and nonhypoxic cellular responses. ${ }^{6}$ These data strongly support the need for appropriately powered randomized trials to determine if treatment of anemia can improve outcome and reduce mortality in our patients. In addition, in order to develop other novel and effective treatment strategies, studies are also required that define the adaptive mechanism(s) that protect against anemia-induced mortality.

Competing interests None declared.

\section{References}

1. Hebert PC, Wells G, Blajchman MA, et al. A multicenter, randomized, controlled clinical trial of transfusion requirements in critical care. Transfusion Requirements in Critical Care Investigators, Canadian Critical Care Trials Group. N Engl J Med 1999; 340: 409-17.

2. Zarychanski R, Houston DS. Anemia of chronic disease: a harmful disorder or an adaptive, beneficial response? CMAJ 2008; 179: 333-7.

3. Anonymous. Multicenter trial of hemodilution in acute ischemic stroke. Results of subgroup analyses. Scandinavian Stroke Study Group. Stroke 1988; 19: 464-71.

4. Musallam KM, Tamim HM, Richards T, et al. Preoperative anaemia and postoperative outcomes in non-cardiac surgery: a retrospective cohort study. Lancet 2011; 378: 1396-407.

5. Hung M, Besser M, Sharples LD, Nair SK, Klein AA. The prevalence and association with transfusion, intensive care unit stay and mortality of pre-operative anaemia in a cohort of cardiac surgery patients. Anaesthesia 2011; 66: 812-8.

6. Tsui AK, Marsden PA, Mazer CD, et al. Priming of hypoxiainducible factor by neuronal nitric oxide synthase is essential for adaptive responses to severe anemia. Proc Natl Acad Sci USA 2011; 108: 17544-9.

Gregory M. T. Hare MD, PhD

The Keenan Research Centre in the Li Ka Shing Knowledge Institute, St. Michael's Hospital, University of Toronto, Toronto, Canada 